\title{
Escala para valororar el estrés en estudiantes de cursos de física en tiempos de covid-19
}

\section{Scale to assess stress in students of physics courses in times of covid-19}

\author{
Mawency Vergel-Ortega ${ }^{1}$ \\ Henry de Jesús Gallardo Pérez ${ }^{2}$ \\ Jorge Fernando Márquez Peñaranda ${ }^{3}$ \\ Universidad Francisco de Paula Santander
}

\section{RESUMEN}

El aprendizaje de la física supone arduos esfuerzos cuyos resultados con frecuencia se muestran en paradójica desproporción lo cual conlleva a generar estrategia para valorar el nivel de estrés entre los estudiantes puesto que esto a su vez, permite establecer acciones pedagógicas y didácticas para la enseñanza de la física y para potenciar el pensamiento físico Objetivo: establecer la validez y fiabilidad de una escala para evaluar el estrés en estudiantes de cursos de física de instituciones educativas de educación superior del municipio de Cúcuta en tiempos de covid-19.

\footnotetext{
$1 \quad$ Licenciada en Matemáticas y Física PhD en Educación. Magister en Gerencia educativa. Universidad Francisco de Paula Santander. Correo. mawencyvergel@ufps. edu.co. Orcid: https://orcid.org/0000-0001-8285-2968 2 Licenciado en matemáticas y Física. PhD en Educación. Magister en Economía. Correo. henrygallardo@ ufps.edu.co. Orcid: https://orcid.org/0000-0003-4377-3903 3 Ingeniero civil. PhD en ingeniería. Universidad Francisco de Paula Santander. Correo. jorgefernandomp@ ufps.edu.co. Orcid: https://orcid.org/0000-0002-9193-8349
}

Materiales y Métodos: Estudio descriptivo de corte transversal, diseño instrumental; evidencia de validez de constructo se evaluó a través de análisis factorial confirmatorio, correlación y comparación; la consistencia interna se estimó mediante el coeficiente alfa de Cronbach. La muestra estuvo constituida por 1200 participantes seleccionados de la población de estudiantes de instituciones educativas públicas y privadas del municipio de Cúcuta, Colombia, de diferentes programas académicos que tienen matriculado cursos de física. Resultados: se conforma una escala con 20 ítems y 3 factores: ansiedad, presión institucional, afrontamiento y se evalúa su fiabilidad y validez para el diagnóstico de estrés en estudiantes de cursos de física. Conclusión: la escala para evaluar el estrés en estudiantes es válida y fiable. 
PALABRAS CLAVE: estrés, escala, validez, aprendizaje, estudiantes

\section{ABSTRACT}

Learning physics involves hard efforts whose results are often paradoxically disproportionate, which leads to generate a strategy to assess the level of stress among students since this in turn allows to establish pedagogical and didactic actions for teaching physics and to enhance physical thinking. Objective: to establish the validity and reliability of a scale to evaluate the stress in students of physics courses in higher education institutions of the municipality of Cúcuta in times of covid-19. Materials and Methods: Descriptive cross-sectional study, instrumental design; evidence of construct validity was evaluated through confirmatory factor analysis, correlation and comparison; internal consistency was estimated through Cronbach's alpha coefficient. The sample was constituted by 1200 participants selected from the population of students of public and private educational institutions of the municipality of Cúcuta, Colombia, of different academic programs that have enrolled physics courses. Results: a scale with 20 items and 3 factors is formed: anxiety, institutional pressure, coping and its reliability and validity for the diagnosis of stress in students of physics courses is evaluated. Conclusion: the scale to evaluate stress in students is valid and reliable

KEYWORDS: stress, scale, validity, learning, students

\section{INTRODUCCIÓN}

La palabra estrés fue extraída del latín stringere "tensar o estirar" y fue empleado para hacer referencia a la opresión, adversidad y dificultad [1], es considerado como un fenómeno adaptativo de los seres humanos que contribuye, buena medida, a su supervivencia
[2]. El estrés afecta órganos y funciones de todo el organismo. Los síntomas más comunes son depresión o ansiedad, dolores de cabeza, insomnio, indigestión, sarpullidos, disfunción sexual, nerviosismo, palpitaciones rápidas, diarrea o estreñimiento [3]. Las fases son alarma, resistencia, agotamiento. El estrés se puede clasificar de la siguiente manera; amoroso, marital, sexual familiar, por duelo, y el estrés académico [4]. Aunque en el entorno académico, las funciones cognitivas más atendidas en los diversos estudios normativos se concentran en la atención, memoria y lenguaje, se contemplan una amplitud de capacidades cognitivas que van desde la fluidez verbal hasta la cognición social, pasando por la percepción, inteligencia, praxias, orientación y funciones ejecutivas.

Como suele ocurrir en esta clase de estudios (normativos), los trabajos han excluido a sujetos con estrés o patologías asociadas que pudiese comprometer el desempeño cognitivo y por lo tanto interferir durante la ejecución de las pruebas. En estudios sobre estrés, Celis et al [5], encontraron mayores niveles de ansiedad en alumnos de primer año que en alumnos de sexto año, además identificaron factores estresores como realizar un examen, la falta de tiempo para cumplir con las tareas académicas, el exceso de carga academia. Barraza [6] señaló que el ingreso, la permanencia y el egreso del alumno a una institución educativa suele ser una experiencia de aprendizaje acompañada regularmente de estrés [7] y se relaciona a factores como trabajos con mayor peso de notas para aprobar la materia y desarrollar un examen final. Es así como las tareas inherentes a la vida universitaria son percibidas como factores estresantes, y va depender de como los alumnos hagan frente a estas demandas. Para Lazarus [8] uno de los principales generadores de estrés para el ser humano es el académico y si no es manejado de manera adecuada puede resultar perjudicial para el alumno con consecuencia de reacción desadaptativa, evitación o la deserción 
académica. Mientras que, para Jerez y Oyarzo [9], existe una alta prevalencia de estrés en los estudiantes del área de la salud, indicando un mayor estrés el género femenino existiendo diferencia por carreras en las evaluaciones de los profesores, fatiga crónica y dolores abdominales. Chiyong y Villacampa [10] utilizaron el inventario sisco del estrés académico y el registro de notas semestrales, los resultados reflejan que el total de los estudiantes en algún momento de su vida universitaria experimentó estrés académico y el nivel de estrés en los estudiantes fue en un nivel promedio. Existen aproximadamente quince dominios vitales relevantes, entre los que se encuentran los siguientes: salud cognitiva, salud económica, salud emocional, salud ambiental, intereses intelectuales, salud comportamental, cuidados de la salud, ocio, satisfacción vital general, actividades no recreativas (incluye trabajo y estudio), salud física, sueno, espiritualidad y sentido, y vitalidad [11].

La presente investigación pretende entonces establecer una escala de estrés para estudiantes de física ante el marco de la pandemia generada por el Covid-19, lo cual ha llevado a trabajo en entornos virtuales $y$, por ende, en casi todas las instituciones de educación superior, a la cancelación o aplazamiento de las prácticas de laboratorio complementarias en su formación. Una vez definida y validada la escala se dejará como base para aplicación en otros estudios relacionados.

El modelo, construido y validado, de tres factores: ansiedad, presión instruccional, afrontamiento, es adecuado para evaluar niveles de estrés en estudiantes de física, pues el desarrollo de actividades académicas en forma virtual genera ansiedad tanto por la carga académica como por la dificultad de realizar actividades empíricas en el laboratorio que faciliten la comprensión y el aprendizaje de los diferentes tópicos de la física junto con el cambio inesperado de metodología de trabajo para estudiantes y docentes.

\section{MÉTODO}

El diseño de la presente investigación es instrumental [12], al analizar validez y fiabilidad de un instrumento de medida psicológica y se siguen los estándares de validación desarrollados conjuntamente por la American Educational Research Association (AERA), American Psychological Association (APA) y el National Council on Measurement in Education (NCME) [13]. El estudio ha contado con un total de 1200 participantes. Los criterios de inclusión fueron: estudiantes que adelantan cursos de física en su formación profesional, residentes en Cúcuta y zona metropolitana, que voluntariamente aceptaran participar mediante el diligenciamiento del consentimiento informado. Todos fueron elegidos intencionalmente debido a su accesibilidad. La mayoría de los jóvenes de nivel sociocultural y económico medio. Se utilizó la versión validada adaptada por Chiyong [14].

El análisis estadístico se realizó mediante etapas. En la primera etapa se analizaron las medidas descriptivas de los ítems y sus características distribucionales. El supuesto de normalidad se evaluó a través de coeficientes de asimetría y curtosis y la prueba Kolmogorov-Smirnov [15], obteniéndose que los resultados siguen distribución normal al nivel de significación del $5 \%$. En la segunda etapa, para identificar validez estructural se realizó un análisis factorial confirmatorio (AFC) para dos modelos: modelo original [16] compuesto por cinco factores y el segundo modelo compuesto por tres factores y 20 ítems (F1 con 9 ítems; F2 con 8 ítems; F3 con 3 ítems). Además, se consideraron pesos factoriales aceptables a partir de 0.40 [17].

El método de estimación empleado fue Weighted Least Square Mean and Variance Adjusted (WLSMV), debido a la naturaleza categórica de las variables de estudio [18]. Se compararon los índices de ajuste de ambos modelos, entre los que destacaban la razón chi cuadrado 
entre los grados de libertad $(\mathrm{x} 2 / \mathrm{gl})$ con valores esperados menores a 3, Root Mean Square Error of Approximation (RMSEA) y Standarized Root Mean Square Residual (SRMR) con valores por debajo a .08 sugeridos por Bentler \& Bonnet [19]. Se incluyó el Comparative Fit Index (CFI) de bondad de ajuste de Jöreskog \& Sörbom [20] para verificar el ajuste incremental, cuyos valores aceptables se encontraban por encima de 0.95 , al igual que el índice de TuckerLewis (TLI) el cual permitió cotejar el ajuste de parsimonia [21]. Adicionalmente, a partir de las cargas factoriales, se calculó la varianza promedio extraída (Average Variance Extracted [AVE]) considerando valores alrededor del 0.50 como satisfactorios, con ello se verificaron las evidencias de validez interna convergente [22]. Las evidencias de fiabilidad por consistencia interna se evaluaron a través del coeficiente omega $(\omega)$, debido a que este coeficiente sigue siendo imparcial con elementos congéneres con errores no correlacionados. Adicionalmente, se calculó el coeficiente de fiabilidad compuesta (compositive reliability [CR]). Para evaluar la validez estructural se realizó un análisis factorial confirmatorio con un estimador robusto (WLSMV), por la naturaleza categórica de las variables [23].

\section{RESULTADOS}

\section{Descripción de los participantes}

Los participantes en el estudio se distribuyen así: $74 \%$ son mujeres y $26 \%$ son hombres, en su mayoría de estrato socio económico medio, sus edades oscilan entre los 18 y 26 años con un promedio de edad de 22.8 años (23.4 años en hombre y 22.6 para mujeres, con desviación estándar conjunta de 3.98 años). Se realiza verificación de similitud estadística entre los dos grupos con respecto a las variables iniciales a fin de garantizar la universalidad de los resultados; para ello se utilizan pruebas de comparación para poblaciones independientes, obteniéndose que no existen diferencias significativas en promedios, varianzas ni en valores proporcionales.

Se utilizó la versión validada adaptada por Chiyong [14], compuesta por 29 ítems escalados en formado Likert de cinco puntos ( 1 = muy bajo, 2 = bajo, $3=$ medio, $4=$ alto, $5=$ muy alto). La mayor puntuación hace referencia a mayor presencia del constructo evaluado. El estudio de validación identificó evidencias de validez estructural a partir de un análisis factorial exploratorio con medida Kaiser- Meyer- Olkin (KMO) de 0.08 Así, a partir de la prueba de esfericidad de Barlett se pudo determinar una matriz de correlaciones positiva $(p<0.01)$, el criterio de autovalor mayor que 1 , explicó el $56 \%$ de la varianza total, esto significa que los factores alcanzaron a explicar la correlación entre ítems.

\section{ANÁLISIS DEL TEST DE CINCO FACTORES}

Análisis descriptivo de los ítems que componen el test para evaluar el estrés académico con 5 factores, los cuales se calcularon a partir de las puntuaciones obtenidas en cada uno de los ítems que conforman el instrumento evidencian que un $16.2 \%$ se ubica en el nivel muy alto, 12.5 $\%$ se ubica en el nivel alto, el $52,5 \%$ se ubica en el nivel promedio, $14.6 \%$ se ubica en nivel bajo y un $4.2 \%$ con un nivel muy bajo. El nivel de estrés académico es muy alto en los estudiantes de tercer semestre $57 \%$ y muy bajo en los del cuarto semestre con un $44.4 \%$. El valor promedio en los ítems oscila entre 3.06 a 3.83 con desviación estándar cuyos valores están entre 1.02 y 1.29, indicando una dispersión relativa inferior al $40 \%$. La prueba K-S indica que la distribución de la puntuación en los ítems está normalmente distribuida.

Respecto a la estructura interna del modelo de cinco factores, se aprecia que alcanza cargas factoriales satisfactorias $(\Lambda>0.40)$ en cada uno de sus factores [18]. Se calculó la varianza promedio extraída (Average Variance Extracted [AVE]) cuyos valores se encuentran 
por debajo de lo esperado (0.50), lo que indica que la estructura original no presenta validez interna convergente [24]. En general, las cargas factoriales de cada factor son inferiores a las covarianzas con otros factores. Así, el factor estresores, presenta covarianzas que fluctúan entre 0.32 (F2); síntomas psicológicos 0.7 (F1), síntomas físicos y síntomas comportamentales alcanza covarianzas de 0.57 (F5); afrontamiento cuyas covarianzas oscilan entre 0.80 y 0.91 (F4); obteniendo una matriz de correlaciones positiva y con coeficientes que fluctúan entre 0.45 y 0.14 . De otra parte, para dimensiones estresores se obtiene un alfa de 0.79 con 8 elementos, síntomas físicos alfa de 0.93 con 6 elementos, síntomas psicológicos 0.12 con 5 elementos, síntomas comportamentales con alfa de 0.37 con elementos y afrontamiento con alfa de 0.75 con 6 elementos. Se encuentra entonces que los índices de fiabilidad son relativamente bajos para el caso de análisis.

\section{ANÁLISIS DEL TEST DE TRES FACTORES}

Dados índices de fiabilidad bajos en el test de cinco factores, se propone la elaboración y validación de un test con tres factores. Para ello se generan cien ítems con base a entrevista aplicada a expertos y resultados de un grupo focal. El test se aplica a los participantes del estudio y los resultados se analizan mediante la aplicación del modelo de teoría de respuesta al ítem llevando a reducir a veinte ítems que se reorganizan en tres factores con base en la fiabilidad de test, saturaciones de los factores y las evidencias sobre cargas factoriales más homogéneas. Se evalúan las cargas factoriales a cada factor y se encuentra que están por arriba de las del modelo anterior $[10,25]$. Las correlaciones entre los factores son positivas, pero inferiores a las covarianzas entre factores.

En el primer modelo, donde se conservan los ítems en su versión original, se determinó que el índice de bondad de ajuste CFI=0.91, índice de Tucker Lewis, TLI $=0.96$, raíz del residuo cuadrático promedio de aproximación RMSEA $=0.065$ no se ajustan apropiadamente [26]. Mientras que, para el segundo modelo, luego de eliminación y reagrupación de ítems, los índices de ajuste alcanzan valores satisfactorios con $X^{2}=1473.94$, RMSEA $=0.065 \quad C F I=0.97$, $\mathrm{TLI}=0.98[10]$.

Tabla 1. Índices de bondad de ajuste de dos modelos del AFC

\begin{tabular}{cccccccc}
\hline Modelo & $\boldsymbol{X 2}$ & $\boldsymbol{p}$ & $\boldsymbol{C F I}$ & $\boldsymbol{T L I}$ & $\boldsymbol{R M S E A}$ & $\boldsymbol{L i}$ & $\boldsymbol{L S}$ \\
\hline 5 factores & 2651.00 & $<0.01$ & 0.91 & 0.96 & 0.065 & 0.062 & 0.068 \\
3 factores & 1259.10 & $<0.01$ & 0.97 & 0.98 & 0.065 & 0.056 & 0.061 \\
\hline
\end{tabular}

En el modelo de tres factores, se establecen las dimensiones reacciones físicas asociadas a ítem de ansiedad, afrontamiento (cognitivo y emocional) y, presión instruccional presentan consistencia interna alta, ya que valores del Alfa de Cronbach oscilan entre $0.933,0.936$ y 0.932 . En cuanto a los demás componentes los valores alfa son "aceptables", oscilando estas entre 0.77 y 0.73; Según George y Mallery [27], estadístico de KMO (Kaiser,Meyer y Olkin), el resultado es de 0.837 , esto refiere una reciprocidad entre los factores considerados en el inventario y por ende, se puede proceder con el análisis factorial. De otra parte, prueba de esfericidad de Bartlett, muestra una probabilidad de $p=0.000$ $<0.05$, luego el modelo factorial es el más adecuado para explicar los datos. la extracción de 3 factores es explicada por el $78.287 \%$ de la varianza, por lo cual, se satisface el criterio de la multidimensionalidad del constructo estrés académico teórica y empíricamente. a través del procedimiento de varimax. Los pesos factoriales son en su totalidad mayores de 0.30 . La pertenencia de un ítem para un determinado 
factor se justifica por la mayor carga del peso factorial; en este sentido, en todos los factores se confirma la correspondencia entre los ítems, se puede apreciar una estructura de tres factores cuyas cargas factoriales se ubican por encima de 0.40 los cuales corresponden a valores adecuados [18].

Tabla 2. Varianza

\begin{tabular}{|c|c|c|c|c|c|c|}
\hline \multirow[b]{2}{*}{ Componente } & \multicolumn{3}{|c|}{ Autovalores iniciales } & \multicolumn{3}{|c|}{$\begin{array}{c}\text { Sumas de las saturaciones al cuadrado } \\
\text { de la extracción }\end{array}$} \\
\hline & Total & $\begin{array}{c}\% \text { de la } \\
\text { varianza }\end{array}$ & $\begin{array}{c}\% \\
\text { acumulado }\end{array}$ & Total & $\begin{array}{c}\% \text { de la } \\
\text { varianza }\end{array}$ & $\begin{array}{c}\% \\
\text { acumulado }\end{array}$ \\
\hline 1 & 2,383 & 51,916 & 51,916 & 2,383 & 51,916 & 51,916 \\
\hline 2 & 1,399 & 19,997 & 71,913 & 1,399 & 19,997 & 71,913 \\
\hline 3 & 1,311 & 7,557 & 79,47 & 1,311 & 7,557 & 79,47 \\
\hline 4 & 1,231 & 6,157 & 85,627 & & & \\
\hline 5 & 1,22 & 5,094 & 90,721 & & & \\
\hline 6 & 1,147 & 2,251 & 92,972 & & & \\
\hline 7 & 1,106 & 1,811 & 94,783 & & & \\
\hline 8 & 1,089 & 1,745 & 96,528 & & & \\
\hline 9 & 0,986 & 1,649 & 98,177 & & & \\
\hline 10 & 0,947 & 1,008 & 99,185 & & & \\
\hline 11 & 0,918 & 0,815 & 100 & & & \\
\hline
\end{tabular}

La consistencia de la medición, en este caso muestra que la escala funciona de manera similar bajo diferentes condiciones, dependientes del mismo instrumento, del tiempo de aplicación del que hace la medición, de los sujetos, de la interacción entre estas fuentes y del error aleatorio puro. El análisis muestra que el test es altamente fiable, con un alfa de Cronbach de 0.84 (escala es homogénea). La consistencia interna del instrumento muestra que los ítems miden el mismo concepto, en este caso "estres". De igual manera, el estadístico por elemento muestra que la eliminación de un ítem bajaría la fiabilidad del test (tabla 2). 


\begin{tabular}{|c|c|c|c|c|}
\hline \multicolumn{5}{|c|}{ Tabla 3. Estadístico total de elementos } \\
\hline $\begin{array}{c}\text { Media de la escala } \\
\text { si se elimina el } \\
\text { elemento }\end{array}$ & $\begin{array}{c}\text { Correlación } \\
\text { elemento-total } \\
\text { corregida }\end{array}$ & $\begin{array}{c}\text { Correlación } \\
\text { múltiple al } \\
\text { cuadrado }\end{array}$ & $\begin{array}{c}\text { Alfa de Cronbach } \\
\text { si se elimina el } \\
\text { elemento }\end{array}$ \\
\hline ítem1 & 58.546 & .176 & .119 & .394 \\
\hline ítem2 & 57.456 & .204 & .143 & .389 \\
\hline ítem3 & 50.514 & -.185 & .108 & .450 \\
\hline ítem4 & 51.163 & -.169 & .161 & .455 \\
\hline ítem5 & 51.350 & .097 & .113 & .408 \\
\hline ítem6 & 51.253 & .154 & .154 & .397 \\
\hline ítem7 & 50.411 & -.185 & .139 & .455 \\
\hline ítem8 & 50.606 & .120 & .138 & .404 \\
\hline ítem9 & 50.471 & .251 & .175 & .379 \\
\hline ítem10 & 50.756 & .135 & .215 & .401 \\
\hline ítem11 & 50.557 & .192 & .147 & .391 \\
\hline ítem12 & 50.734 & -.189 & .109 & .458 \\
\hline ítem13 & 51.031 & .064 & .121 & .414 \\
\hline ítem14 & 50.654 & -.199 & .135 & .459 \\
\hline ítem15 & 50.960 & -.162 & .121 & .456 \\
\hline ítem16 & 50.934 & .248 & .202 & .376 \\
\hline item17 & 50.531 & .210 & .152 & .386 \\
\hline ítem18 & 50.974 & -.036 & .213 & .434 \\
\hline item19 & 50.509 & .245 & .207 & .381 \\
\hline ítem20 & 50.966 & .194 & .129 & .390 \\
\hline
\end{tabular}

Los ítems dentro de la escala se correlacionan o son complementarios el uno al otro, la escala es unidimensional lo que quiere decir que mide un solo concepto: estrés. Al eliminar ítems el alfa disminuye, con lo cual se considera para este caso 20 ítems como dimensionalidad del conjunto de ítems. Así mismo, alfa basada en elementos estandarizados muestra alta homogeneidad y fiabilidad del instrumento (Tabla 3). El nivel de covariación de los ítems entre sí y la cantidad de ítems o partes que conforman la prueba (Tabla 4).

\begin{tabular}{|c|c|c|c|c|c|c|c|}
\hline & & & $\begin{array}{l}\text { Suma de } \\
\text { cuadrados }\end{array}$ & gl & $\begin{array}{c}\text { Media } \\
\text { cuadrática }\end{array}$ & $\begin{array}{c}\text { Chi- } \\
\text { cuadrado } \\
\text { de } \\
\text { Friedman }\end{array}$ & Sig. \\
\hline \multicolumn{3}{|c|}{ Inter-personas } & 1170,239 & 1198 & 3,359 & & \\
\hline \multirow{5}{*}{$\begin{array}{l}\text { Intra- } \\
\text { personas }\end{array}$} & \multicolumn{2}{|c|}{ Inter-elementos } & 745,377 & 19 & 27,607 & 14,070 & ,000 \\
\hline & \multirow{3}{*}{ Residual } & $\begin{array}{c}\text { No } \\
\text { aditividad }\end{array}$ & $28,831^{a}$ & 1 & 28,831 & 14,715 & $\overline{, 000}$ \\
\hline & & Equilibrio & 18460,327 & 9422 & 1,959 & & \\
\hline & & Total & 18489,158 & 9423 & 1,962 & & \\
\hline & \multicolumn{2}{|c|}{ Total } & 19234,536 & 9450 & 2,035 & & \\
\hline \multicolumn{3}{|c|}{ Total } & 20406,775 & 9799 & 2,083 & & \\
\hline
\end{tabular}


Se puede decir que la confiabilidad mide el error que puede generar un instrumento al ser inestable y aplicarse en diferentes ocasiones. El coeficiente de correlación 0.84 muestra estabilidad temporal y consistencia en las respuestas de los individuos (Tabla 4).

\begin{tabular}{|c|c|c|c|c|c|c|c|}
\hline & $\begin{array}{c}\text { Correlación }^{\text {intraclase }}{ }^{\mathbf{b}} \\
\end{array}$ & \multicolumn{2}{|c|}{$\begin{array}{c}\text { Intervalo de } \\
\text { confianza 95 \% }\end{array}$} & \multicolumn{2}{|c|}{ Prueba F con valor verdadero 0 } \\
\cline { 3 - 8 } & $\begin{array}{c}\text { Límite } \\
\text { inferior }\end{array}$ & $\begin{array}{c}\text { Límite } \\
\text { superior }\end{array}$ & Valor & $\mathbf{g l 1}$ & $\mathbf{g l 2}$ & Sig. \\
\hline $\begin{array}{c}\text { Medidas } \\
\text { individuales } \\
\text { Medidas } \\
\text { promedio }\end{array}$ &, $84025^{\mathrm{a}}$ &, 017 &, 035 & 1,712 & 1197 & 1197 &, 000 \\
\hline
\end{tabular}

Nota: Modelo de efectos mixtos de dos factores en el que los efectos de las personas son aleatorios y los efectos de las medidas son fijos. a. El estimador es el mismo, ya esté presente o no el efecto de interacción. b. Coeficientes de correlación intraclase de tipo $C$ utilizando una definición de coherencia, la varianza inter-medidas se excluye de la varianza del denominador. c. Está estimación se calcula asumiendo que no está presente el efecto de interacción, ya que de otra manera no es estimable.

Fuente: elaboración propia.

La estabilidad del instrumento se analizó a través de la escala de Gutman. Se observa un coeficiente de reproductibilidad $\mathrm{CR}=0.84$, escala de Guttman cercana Alfa de Cronbach mostrando fiabilidad y estabilidad del instrumento para factor 1 lambda $=0.977$, para factor 2 lambda $=0.99$ y para factor 3 Lambda $=0.989$. La reproductibilidad marginal mínima es 0.977 efecto de reactivos que se distribuyen con valor modal extremo, el coeficiente de escalabilidad de 0.174 , es decir, es una escala no acumulativa, no siendo necesario el que cada individuo tenga una escala específica, mostrando reproductibilidad del instrumento para toma de decisiones en conjunto de todos los ítems por factor. Lo anterior muestra que el instrumento es estable, es decir, se obtienen los mismos resultados al utilizar el instrumento en condiciones similares en diferentes ocasiones y es factible generalizar resultados. El coeficiente de correlación es superior a 0.6, mostrando fiabilidad aceptable para medidas únicas y alta para medidas promedio (Tabla 4). En precisión, la validez a través de jueces muestra que el instrumento mide la realidad en torno a estrés. Así para grupos contrastantes jueces para 5 jueces; una vez realizada la evaluación se insertaron las respuestas de evaluadores determinando cada coeficiente de acuerdo a los evaluadores (Tabla 5), donde para cada cuadro se observa que el valor de Kappa es estadísticamente significativo diferente de cero, y teniendo en cuenta su valoración [28], su valor Kappa $(0,795)$ sugiere que las calificaciones de los expertos son similares, existiendo un acuerdo fuerte casi perfecto entre ellos. Con lo cual se concluye que la escala es válida. 
Tabla 5. Matrix de acuerdo Kappa

\begin{tabular}{|c|c|c|c|c|c|c|c|c|}
\multicolumn{7}{|c|}{ Tabla 5. Matrix de acuerdo Kappa } & \\
\hline & E1 & E2 & E3 & E4 & E5 & & \\
\hline E1 & 1 & 0,8 & 0,88 & 0,92 & 0,87 & 0,8675 & & \\
\hline E2 & & 1 & 0,95 & 0,938 & 0,983 & 0,957 & & \\
\hline E3 & & & 1 & 0,799 & 0,638 & 0,718 & & \\
\hline E4 & & & & 1 & 0,638 & 0,638 & & \\
\hline E5 & & & & & 1 & & & \\
\hline & & & & & & & 0,795 \\
\hline
\end{tabular}

\section{CONCLUSIONES}

El modelo de tres factores: ansiedad, presión instruccional, afrontamiento, es adecuado para evaluar niveles de estrés en estudiantes de física, pues el desarrollo de actividades académicas en forma virtual genera ansiedad entre los estudiantes tanto por la carga académica como por la dificultad de realizar actividades empíricas en el laboratorio que faciliten la comprensión y el aprendizaje de los diferentes tópicos de la física. El afrontamiento resulta como una respuesta adaptativa para reducir el estrés causado por la carga emocional del estudiante por su uso excesivo de recursos en la comprensión de fenómenos físicos realizada desde su propia percepción, ya que el aprendizaje colectivo y las prácticas de laboratorio no son aplicables completamente en los procesos de enseñanza y de aprendizaje.

El estudio muestra que los estudiantes en el desarrollo de los cursos de física, como fundamento básico y complementario a su formación profesional, presentan niveles de estrés durante su estancia académica en la institución, en especial en tiempos de covid, cuando la mayor parte del trabajo académico se realiza en forma virtual.

La evidencia empírica demuestra que el constructo estudiado, estrés está representado en una estructura interna de tres factores, reafirmando su concepto como un proceso dinámico, complejo y multidimensional que considera a sus factores latentes y a sus respectivas variables (ítems). Los resultados hallados indican que test ajustado para evaluar el estrés en estudiantes en tiempos de Covid aplicado en estudiantes de física en cursos universitarios cuenta con adecuadas propiedades psicométricas que permiten considerarlo como una medida válida y confiable que puede ser empleada en investigaciones futuras como instrumento de medición de la calidad de vida.

La escala obtenida es posible migrarla para análisis en el tiempo y para extrapolarla a otras disciplinas en las cuales los factores determinantes del estrés en estudiantes sean similares a los estudiantes universitarios de cursos de física.

\section{REFERENCIAS BIBLIOGRÁFICAS}

[1] Molina T Gutiérrez A Hernández L \& Contreras 2008 Estrés psicosocial: Algunos aspectos clínicos experimentales Anales de Psicología, 24(2) 353

[2] Peiró J y Salvador A 1993 Control del estrés laboral (España: Editorial UDEMA S. A)

[3] Rojas G Chang S y Delgado L 2015 Niveles de estrés y formas de afrontamiento en estudiantes de Medicina en comparación con estudiantes de otras escuelas. Revista Dialnet 151(4) 443

[4] Orlandini A 1999 El estrés. Qué es y cómo evitarlo. (México: Fondo de Cultura Económica) 
[5] Celis C Bustamante M Cabrera D Cabrera M Alarcón W y Monge E 2001 Ansiedad y Estrés Académico en Estudiantes de Medicina Humana del Primer y Sexto Año. Revista anuales de la universidad Mayor de San Marcos 62(1) 25

[6] Barraza A 2008 El estrés académico en alumnos de maestría y sus variables moduladoras: Un diseño de diferencias de grupo Avances en Psicología Latinoamericana 26(2) 270

[7] Boullosa G 2013 Estrés académico y afrontamiento en un grupo de estudiantes de una universidad privada de Lima Tesis para optar el título de Licenciada en Psicología. Universidad Pontificia La Católica

[8] Lazarus R \& Folkman S 1986 Estrés y procesos cognitivos (Barcelona: Martínez Roca)

[9] Jerez M y Oyarzo C 2015 Estrés académico en estudiantes del Departamento de Salud de la Universidad de Los Lagos Osorno Revista Chilena de neuro-psiquiatría 53149

[10] Chiyong T y Villacampa S 2015 Estrés y desempeño académico en estudiantes de odontologia. departamento académico de estomatología preventiva y social de la facultad de odontología de la Universidad Nacional Mayor de San Marcos 18(1) 23

[11] Connor K \& Davidson J 2003 Development of a new resilience scale: The Connor Davidson Resilience Scale (CD-RISC) Depression and Anxiety 18(2) 76

[12] Vergel M, Gómez C S y Rojas-Suarez J 2020 Lean seis sigma en la gestión de la vicerrectoría de bienestar universitario (Bogotá: ECOE)

[13] Gallardo H Vergel M y Gómez C 2018 Modelo estructural para evaluar el estrés en profesionales que orientan a la niñez en programas de formación inclusiva Logos Ciencia \& Tecnología 10(2) 120

[14] Rueda-Archila N Romero-Pérez D y GómezVergel CS 2019 Factores que inciden en la aplicación de números racionales en educación básica secundaria en una zona de frontera Aibi revista de investigación, administración e ingeniería, 16

[15] Pérez A 1999 Dependencia, cuidado informal y función familiar. Análisis a través del modelo sociocultural de estrés y afrontamiento Tesis doctoral (Salamanca: Universidad de Salamanca)

[16] Vergel-Ortega M y Parra H M 2013 Modelo para evaluar la pertinencia social en la oferta académica de la Universidad Francisco de Paula Santander Revista de investigación en administración e ingeniería 3(1) 3

[17] Vergel M Rincón O y Zafra L 2017 Modelo de calidad servqual para evaluar factores que influyen en el uso de blogs como recurso didáctico del cálculo (Bogotá: Grupo Editorial Ibañez-Uniediciones)

[18] Stahl B y Goldstein E 2013 Mindfulness para reducir el estrés. Una Guía Práctica. (Barcelona: Editorial Kairós)

[19] Bentler P and Bonnet D 1980 significance tests and goodness of fit in the analysis of covariance structures Psychological Bulletin 88(3) 588

[20] Jöreskog K 1978 Structural analysis of covariance and correlation matrices. Psychometrika 36409

[21] Santiago M Vergel M y Gallardo H Resiliencia en estudiantes exitosos en matemáticas Praxis \& Saber 11(26) e9973 
[22] Domínguez-Lara S 2016 Secretos del coeficiente alfa Actas Urológicas Españolas 40(7) 471

[23] Naranjo M 2009 Una revisión teórica sobre el estrés y algunos aspectos relevantes de éste en el ámbito educativo Revista Educación 33(2) 171

[24] Maldonado-Carvajal S Gómez C y Vergel M 2020 Impacto del programa jóvenes en acción en la formación inicial de educadores en matemáticas Revista Boletín Redipe 9(7) 165

[25] Monzón M 2007 Estrés académico en estudiantes universitarios Revista Apuntes de Psicología 25(1) 87

[26] Fortich N 2013 Prevalencia del estrés académico, factores asociados y niveles de presión arterial en estudiantes de pregrado de odontología de la corporación universitaria Rafael Núñez de Cartagena. Tesis inédita de Maestría. (Cartagena: Corporación universitaria Rafael Núñez)

[27] Pidgeon A \& Pickett L 2017 Examining the differences between university students' levels of resilience on mindfulness, psychological distress and coping strategies. European Scientific Journal 13(12) 103

[28] Fu C Leoutsakos J \& Underwood C 2014 An examination of resilience cross-culturally in child and adolescent survivors of the 2008 China earthquake using the ConnorDavidson Resilience Scale (CD-RISC) Journal of Affective Disorders 155149 